\title{
$\underline{\mathbf{P}-211}$
}

\section{Synthesis of Substituted Bis(indolyl)methanes by Using Sodium Thiosulfate in Polyethylene Glycol 400 (PEG-400)}

\author{
Muhammad Taha ${ }^{1, *}$, Nor Hadiani Ismail ${ }^{2}$, Faridahanim Mohd Jaafar ${ }^{2}$ and Khalid Mohammed Khan ${ }^{3}$ \\ ${ }^{1}$ Atta-ur-Rahman Institute for Natural Product Discovery, Universiti Teknologi MARA (UiTM), Puncak Alam Campus, \\ 42300 Bandar Puncak Alam, Selangor, Malaysia; ${ }^{2}$ Faculty of Applied Science UiTM, 40450 Shah Alam, Selangor, \\ Malaysia; ${ }^{3}$ H.E.J. Research Institute of Chemistry, International Center for Chemical and Biological Sciences, University \\ of Karachi, Karachi-75270,Pakistan; E-mail: taha_hej@yahoo.com; muhamm9000@puncakalam.uitm.edu.my
}

A large number of biologically active natural products contain indole as a part of their structures, Numerous bis(indolyl)methane have been isolated from several terrestrial and marine plants, possessing a variety of biological activities. Nevertheless, the discovery of potent anti-carcinogenic bis(indolyl)methane have attracted a major attention of researchers. Bis(indolyl)methane is the most active cruciferous substance for promoting beneficial estrogen metabolism in women and men, and may have useful application as breast cancer preventive agent. Different indoles were reacted with different aldehydes in the presence of equimolar amount of sodium thiosulfate in PEG 400 yielded corresponding substituted bis(indolyl)methanes in good yields. We tested same reaction with different solvents but we found that the PEG gave the desire reaction yield in short reaction time. This is a facile, environmentally friendly and cheap method towards the synthesis of an important class of organic compounds. All synthesized compounds are characterized by art of spectroscopy.

Keywords: Bis(indolyl)methanes, sodium thiosulfate, PEG400. 